\title{
La edición y la literatura como campos sobrepuestos. Breve apunte sobre su estudio ${ }^{*}$
}

\author{
Publishing and literature as superposed fields. \\ Brief note on their analysis
}

\begin{tabular}{c} 
NuNO MEDEIROs $^{* *}$ \\
\hline $\begin{array}{c}\text { Universidade Nova de Lisboa. Lisboa, Portugal } \\
\text { nuno.medeiros@fcsh.unl.pt }\end{array}$
\end{tabular}

T a edición literaria ha constituido un objeto privilegiado en el ámbito de la producción sociológica e historiográfica de las prácticas editoriales. Pensar el libro editado tiende, a menudo, a la reducción de pensarlo en el presupuesto del género literario, forma históricamente naturalizada de su asimilación al soporte impreso (Nunes, 1996; 2002). En este sentido, no pocas veces la investigación parece funcionar como un espejo de la correlación de fuerzas existente en el marco valorativo del campo editorial, que corona la sobrelegitimidad del libro literario frente a los restantes géneros. Este atributo de la representación editorial dificulta sobremanera la ambición de pensar el universo de la edición de libros a partir de generalizaciones acuñadas científicamente (Coser, Kadushin y Powell, 1982). El volumen colectivo dirigido por Olivier Bessard-Banquy (2006) es un excelente ejemplo de cómo el discurso académico puede no solamente reproducir las percepciones vigentes en amplios sectores del mundo editorial, que propugnan una ligazón automática y garante de respetabilidad entre edición y literatura, sino también reforzar su legitimidad. La obra citada basa toda su construcción

* Le agradezco a Fátima Ribeiro de Medeiros los comentarios que hizo al presente artículo, que contribuyeron a mejorarlo. Agradezco asimismo el apoyo concedido por la Escola Superior de Tecnologia da Saúde del Instituto Politécnico de Lisboa a la materialización de este artículo.

${ }^{* *}$ Sociólogo. Profesor de sociología en la Escola Superior de Tecnologia da Saúde de Lisboa del Instituto Politécnico de Lisboa e Investigador de ciencias sociales e históricas en la Universidade Nova de Lisboa, Faculdade de Ciências Sociais e Humanas. 
analítica en la importancia simbólica de la edición literaria en relación a la mayor parte de los otros dominios de género editorial. La posición cimera de la literatura en el espacio simbólico del mercado del libro, que tiene su correlato en el primer lugar que ocupa en el número de títulos publicados, no corresponde sin embargo a su relevancia económica en el sector editorial, inferior a otros géneros, como el libro escolar o técnico, cuyas cifras de ventas superan significativamente las del segmento literario (Lacy, 1963).

No es muy difícil encontrar documentación testimonial, y su eco académico, concerniente a la asociación establecida entre carreras editoriales y motivaciones que encuentran su razón de ser en la literatura, elevada a escaparate principal de la edición o representada como designio de buena parte de quienes aspiran al destino profesional de editores. Según Jean-Yves Mollier (2000), es posible trazar la génesis de la impostura de considerar lo literario como la esencia misma de la producción de libros -imagen preponderante que se impuso de forma abrupta en los diversos terrenos de las prácticas discursivas, incluyendo el de la investigación - en los cambios ocurridos en la segunda mitad del siglo XVIII, momento crucial de sustitución de la lógica que hacía depender la producción librera de las necesidades sociales entendidas en cada circunstancia por la de radicar en el lado de la oferta el lanzamiento de productos nuevos, susceptibles de generar y animar una demanda: libros prácticos, guías de viaje, diccionarios, manuales escolares, series de ficción de carácter folletinesco y a precio reducido (Villar, 2002).

De resto, la historia y la sociología literarias encuentran en su narrativa la historia y la sociología de la edición. Obras y autores de menor notoriedad podrán deber esa condición, o su perpetuación, a la esfera editorial que les niega la posibilidad de publicación o reedición. Inversamente, la exhumación de nombres olvidados puede deberse a un estímulo editorial, con la edición de obras e incluso géneros que habían sucumbido al peso de los archivos. En otras ocasiones, la ausencia de una edición o reedición en particular, y ello incluso para un libro con mercado asegurado, se debe a motivaciones extraliterarias, situación que ilustra bien el caso de una editorial que posee los derechos de ese libro y atraviesa una coyuntura de dificultades (Bragança, 1999).

El vínculo entre los campos disciplinares mencionados opera asimismo por la investigación sobre la participación editorial en la evolución de los sistemas de legitimidad y consagración y en el fin del monopolio de definición del valor literario, restringido hasta entonces a espacios sociales como el salón o la academia. La posición que la edición pasa a ocupar en el siglo XX como instancia central, a la par de las tradicionales, en la legitimación 
cultural se efectúa en gran medida por el marchamo de prestigio o especialización asociado al nombre de ciertas editoriales (Bassy, 1991). El ejemplo de los premios literarios es tan sólo uno de los indicadores de que la transformación y heterogeneización de los mecanismos sancionadores de la literatura se cruzan profundamente con el ascenso simbólico del editor como descubridor, cuya imagen es moldeada por los autores que edita, configurando él mismo el mercado y el campo de la literatura (Boschetti, 1991; Anderson, 1996). Por otro lado, la articulación entre literatura y edición como procesos con progresiva superposición, se detecta en la acción de los grupos de vanguardia literaria. La afirmación de las heterodoxias o de las designadas posturas rupturistas puede realizarse por medio de la publicación como forma de marcar una posición autónoma ante las audiencias potenciales, viniendo más tarde a sustituir o a añadirse a las posiciones ocupadas por la ortodoxia. Se inauguran, de este modo, revistas que consustancian el sentimiento de comunidad y proyectan su existencia hacia el exterior a través de la creación de una imagen editada de escuela o de comunión estética (Andrade, 2003), permitiendo al mismo tiempo condiciones de libertad creativa a través de la autonomía que se conquista ante el marco de limitaciones económicas -o políticas, en el caso de regímenes de naturaleza autoritaria, como el Estado Novo portugués (Medeiros, 2010) o el franquismo en España (Pericay, Trapiello y Pla, 2002)-, a las que por norma se encuentran sometidas las casas de edición en su lógica de funcionamiento. No es inusual tampoco la transición de la revista a la actividad editorial más institucionalizada (Parinet y Tesnière, 1991).

En ocasiones, el éxito de las escrituras marginales y el correlativo proceso de su rescate de la recepción especializada e iniciática, necesariamente escasa, en dirección al centro legítimo y dominante y al éxito comercial, es el resultado del encuentro entre escritor y editor que comparten una situación de marginalidad. En estos casos, editor y autor comprenden la posibilidad de obtener beneficios mutuos, concurriendo el alza de uno al éxito del otro en una suma positiva que puede determinar la noción de que editor y autor restituirán parte de sí mismos a los respectivos universos que habitan. LouisFerdinand Céline y Robert Denöel representan una conjunción ejemplar de desposeídos, compartiendo la marginalidad y la ausencia de recursos sociales y simbólicos en los respectivos mundos. Su asociación produjo beneficios también repartidos. Denöel, personaje extraño al centro y pobre en capacidad de atracción de la escritura normativa e instalada, se convirtió en la alegoría del editor anticonformista, infundiendo en el nombre de Céline, unánimemente rechazado por sus posiciones ideológicas antisemitas, la res- 
piración de lo sublime-literario paradójicamente fundida en el escándalo profanador. Defendiendo a su autor, el editor marcó una posición y concurrió a su mutación en efigie (Boschetti, 1991).

Es al autor, como figura fuertemente investida de carga simbólica aliada a la relación -conflictiva o armoniosa-con su(s) editor(es) como par que labora en la negación de lo económico (Bourdieu, 1994) y en la apología de la pureza del producto intelectual y cultural, que cabe gran parte de la responsabilidad de la representación ampliamente aceptada y reproducida que asimila la edición de libros al género literario. Este factor conspira, en el entendimiento de Bertrand Legendre (2009), con otros dos en la explicación de esta vinculación que tiende a restringir el acto editorial al dato literario: la atención mediática que la edición literaria recibe, por contraste con otros géneros editoriales, y los usos y prácticas de lectura que se diferencian justamente según las categorías editoriales, normalmente más transversales en el mercado de libros literarios. Construida por un conjunto de prácticas, discursos, consumos y posicionamientos en relación a un campo simbólico y a un mercado encuadrado mediáticamente de modo particular, la idea de que la realidad de la edición se reduce al fenómeno literario acaba por constituir la expresión de un conjunto de procesos de filtraje de esta realidad, de ella construyendo socialmente una percepción conforme con los desiderátums y las estrategias de un número de agentes con intereses específicos en ese campo y en ese mercado (Medeiros, 2009), ya sean esos intereses suscitados por la necesidad de edificar una reputación artística o de ampliar el espectro de potenciales compradores del libro (Bourdieu, 1977; 1992).

En este cuadro, aquí apenas esbozado, la coligación entre edición y literatura demuestra una intervención, o incluso configuración, mutua entre diversos dominios de la vida social. El estudio que pretenda analizar la literatura o la edición de libros desde el punto de vista de la historia o de la sociología no puede abdicar de explorar esta relación. Si, por un lado, el campo literario es el escenario por excelencia, o así ha sido considerado, donde medra en gran medida la identidad del editor y donde mucho de lo que es y fue la actividad editorial encuentra legitimidad y sentido; por otro, la eficacia de la prescripción editorial interfiere en los contornos que el campo literario va adquiriendo, siendo el efecto de mediación ordenadora que los mundos sociales de la edición de libros producen de modo estratégico a partir de posiciones simbólicas y de mercado logradas o deseadas. 


\section{BIBLIOGRAFÍA}

Anderson, Danny. 1996. "Creating cultural prestige: Editorial Joaquín Mortiz", en Latin American Research Review 31 (2), pp. 3-41.

Andrade, Luis Crespo de. 2003. "Introdução", en VV. AA. Revistas: ideias e doutrinas. Leituras do pensamento contemporâneo, pp. 11-18, Lisboa: Livros, Horizonte.

Bassy, Alain-Marie. 1991. "L'édition en marche", en Roger Chartier y Henri-Jean Martin, ed. Histoire de l'édition française, vol. 4, Le livre concurrencée: 1900-1950, pp. 615-620, Paris: Promodis/Fayard.

Bessard-Banquy, Olivier, ed. 2006. L'édition littéraire aujourd'hui, Bordeaux: Presses Universitaires de Bordeaux.

Boschetti, Anna. 1991. "Légitimité littéraire et stratégies éditoriales", en Roger Chartier y Henri-Jean Martin, ed. Histoire de l'édition française, vol. 4, Le livre concurrencée: 1900-1950, pp. 480-527, Paris: Promodis/ Fayard.

Bourdieu, Pierre. 1977. "La production de la croyance: contribution à une économie des biens symboliques", en Actes de la Recherche en Sciences Sociales 13, pp. 3-43.

Bourdieu, Pierre. 1992. Les règles de l'art: genèse et structure du champ littéraire, Paris: Seuil.

Bourdieu, Pierre. 1994. Raisons pratiques: sur la théorie de l'action, Paris: Seuil.

Bragança, Aníbal. 1999. "Revisões e provas. Notas para a história editorial de Os Sertôes de Euclydes da Cunha: as edições Francisco Alves", en Revista de História das Ideias 20, pp. 337-352.

Coser, Lewis; Kadushin, Charles; Powell, Walter. 1982. Books: the culture and commerce of publishing, New York: Basic Books.

Lacy, Dan. 1963. "The economics of publishing, or Adam Smith and literature", en Daedalus 92 (1), pp. 47-48.

Legendre, Bertrand. 2009. L'édition, Paris: Le Cavalier Bleu.

Medeiros, Nuno. 2009. "Acções prescritivas e estratégicas: a edição como espaço social”, en Revista Crítica de Ciências Sociais 85, pp. 131-146.

Medeiros, Nuno. 2010. Edição e editores: o mundo do livro em Portugal, 1940-1970, Lisboa: Imprensa de Ciências Sociais.

Mollier, Jean-Yves. 2000. "L'évolution du système éditorial français depuis l'Encyclopédie de Diderot”, en Jean-Yves Mollier, ed. Où va le livre?, pp. 19-33, Paris: La Dispute. 
Nunes, João Arriscado. 1996. "Fronteiras, hibridismo e mediatização", en Revista Critica de Ciências Sociais 45, pp. 35-71.

Nunes, João Arriscado. 2002. "Materialidade(s) do(s) texto(s) e práticas culturais", en Revista de Comunicação e Linguagens, junio, pp. 393-400.

Parinet, Élisabeth; Tesnière, Valérie. 1991. "Une entreprise: la maison d'édition", en Roger Chartier y Henri-Jean Martin, ed. Histoire de l'édition française, vol. 4, Le livre concurrencée: 1900-1950, pp. 131-148, Paris: Promodis/Fayard.

Pericay, Xavier; Trapiello, Andrés; Pla, Xavier. 2002. Tiempo de editores: Historia de la edición en España 1939-1975, Barcelona: Destino.

Villar, Jorge. 2002. Las edades del libro. Una crónica de la edición mundial, Madrid: Debate. 